\title{
Cyclosporine $A$ and adalimumab change the expression profile of mRNAs and miRNAs related with the histaminergic system in keratinocytes exposed to LPS
}

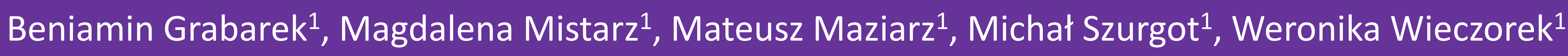

1. Department of Histology, cytophysiology and embryology, Faculty of Medicine in Zabrze, University of Technology in Katowice, Poland

\section{Background:}

In the therapy of psoriasis, anti-TNF drugs and cyclosporine A are used. The histaminergic system is associated with inflammation occurring during psoriasis.

Aim:

The aim of this study was to evaluate the changes in the expression of genes related with the histaminergic system and miRNAs regulating their expression in keratinocytes exposed to LPS and afterwards to adalimumab or cyclosporine A.

\section{Material and methods;}

HaCaT were exposed to $1 \mathrm{ng} / \mathrm{ml}$ of LPS in order to induce inflammation and afterwards were incubated with adalimumab (8 $\mu \mathrm{g} / \mathrm{ml}$ ) or cyclosporine A (100 $\mathrm{ng} / \mathrm{ml})$ for 2,24 and 48 hours, afterwards being compared to untreated cells. To determine differences in the expression pattern of genes associated with a histaminergic system, microarray, RTqPCR and ELISA techniques were used.

\section{Results:}

Out of 22277 mRNAs, 65 mRNAs are related with the histaminergic system. The analysis of the microarray profile indicated that the highest changes (Fold change, $-3.0<\mathrm{FC}>$ +3.0) were observed for HaCaT+LPS: HRH1-3; GABRB3; HNMT; EDN1; ENDRA, FOR HaCaT+LPS+adalimumab: HRH1-3; HNMT; HTR6; GABRB 1 and 3; EDN1; EDNRA; ADA, and for HaCaT+LPS+cyclosporine A: HRH 1-3; HNMT; DRD2; EDN1; GABRB 2 and 3; ADA; ADCYAP1. RTqPCR confirmed the results obtained from a microarray analysis. The strongest relationship with the histaminergic system was observed for the following miRNA: miR-1231; miR-1274; miR-16; and miR-3162. The ELISA results suggested the influence of miRNAs on the expression of selected mRNAs.
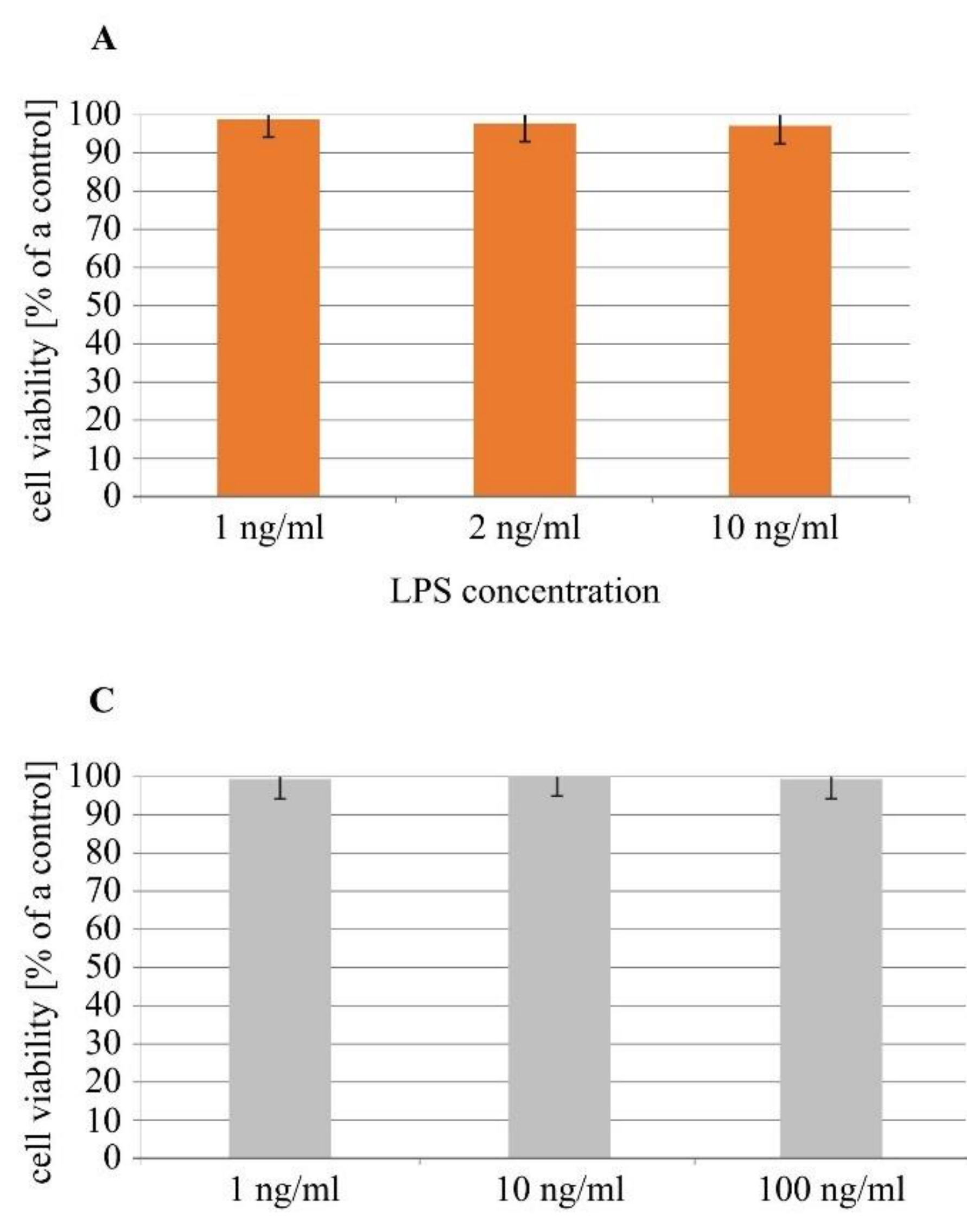

Cyclosporine A concentration

Fig. 1. Results of cytotoxicity assay (A- HaCaT with LPS, B- HaCaT with adalimumab C- HaCaT with cyclosporine A, D- HaCaT with LPS and adalimumab or cyclosporine A)

Fig. 3

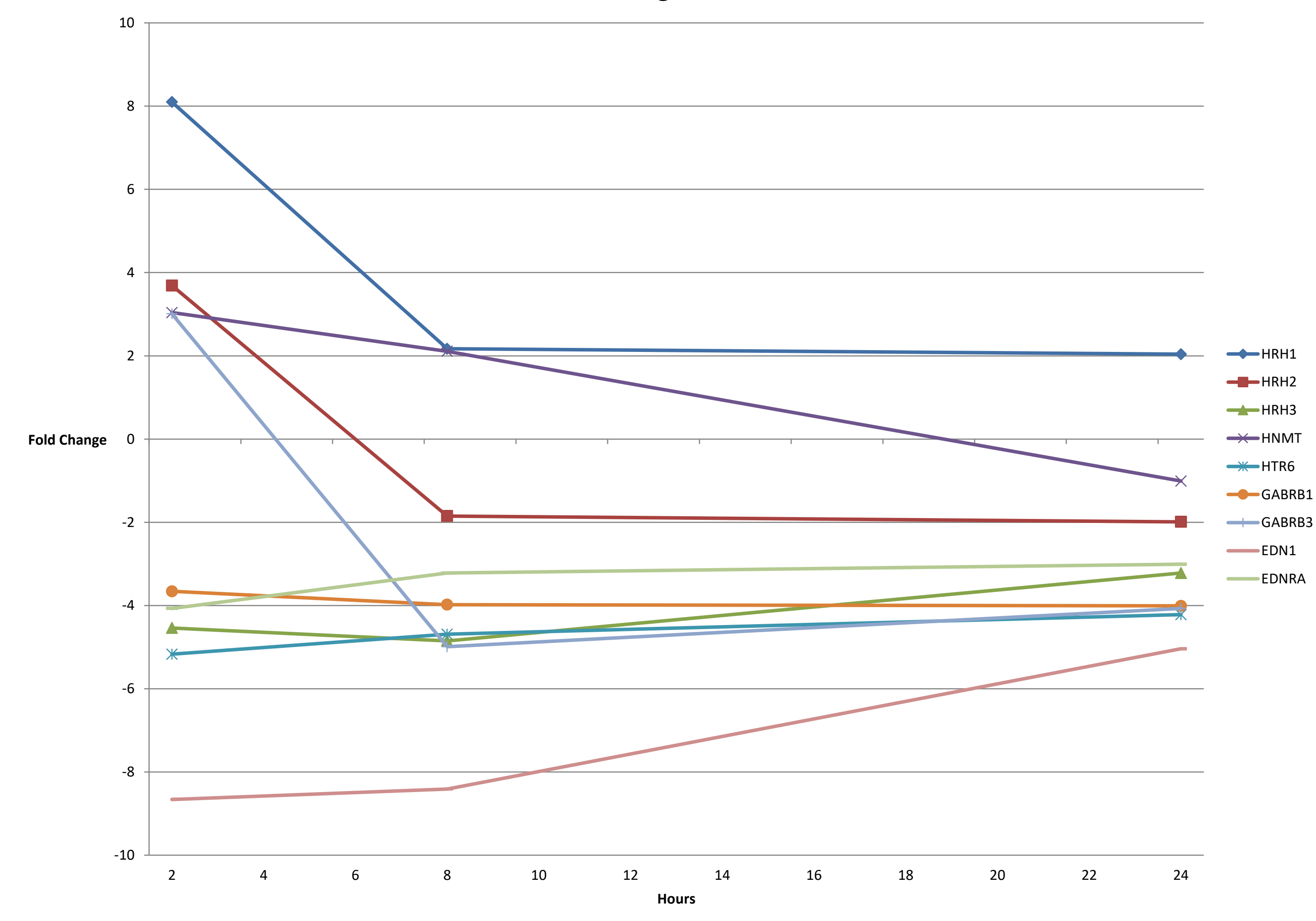

Fig. 3. Microarray results of expression profile of selected genes related to the histminergic system in HaCaT cells treated with HaCaT + LPS + adalimumab
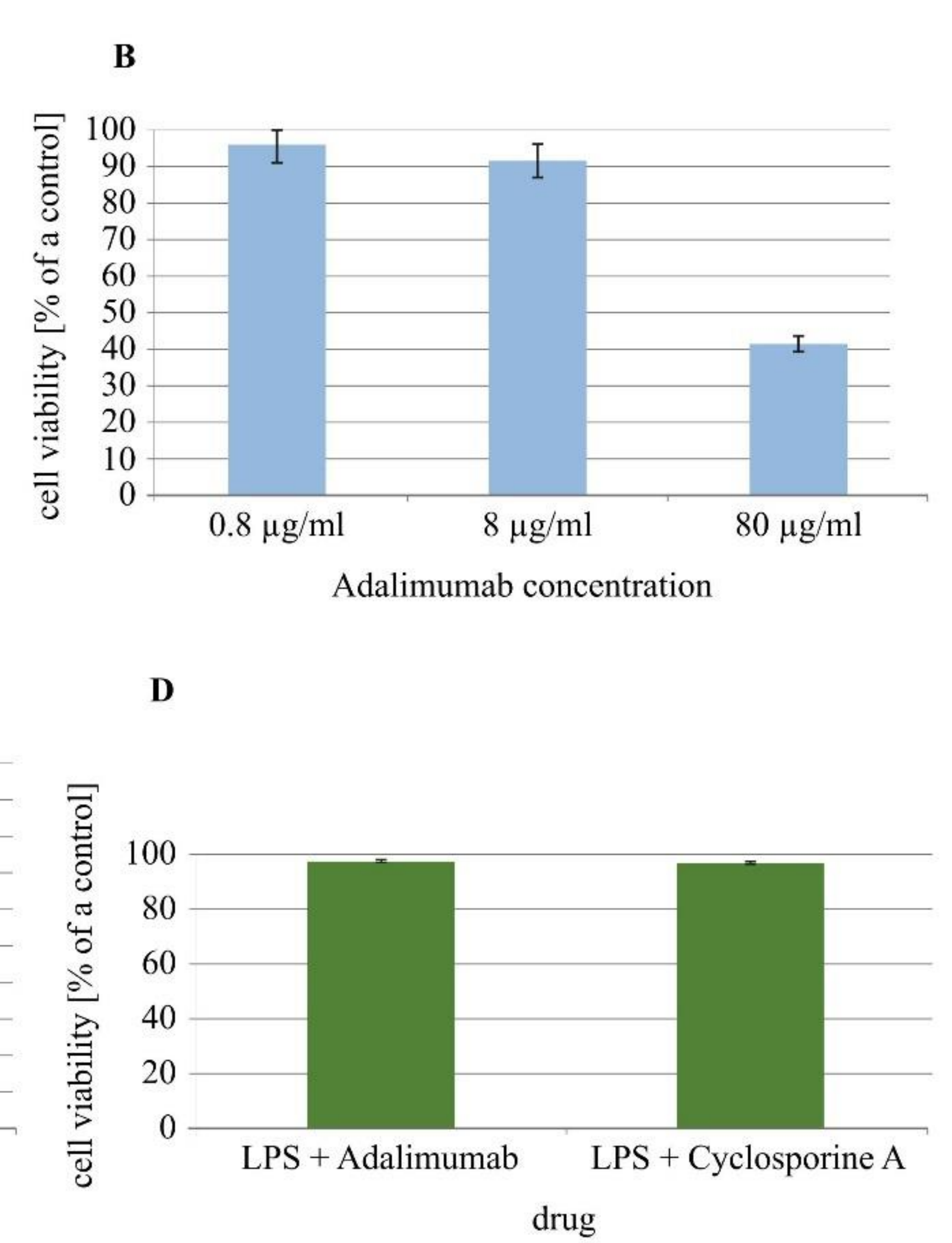
. 